\title{
Measuring the density structure of an accretion hot spot
}

https://doi.org/10.1038/s41586-021-03751-5

Received: 4 March 2021

Accepted: 21 June 2021

Published online: 1 September 2021

\section{Open access}

Check for updates

\author{
C. C. Espaillat ${ }^{1 凶}$, C. E. Robinson ${ }^{2}$, M. M. Romanova ${ }^{3}$, T. Thanathibodee ${ }^{4}$, J. Wendeborn", \\ N. Calvet ${ }^{4}$, M. Reynolds ${ }^{4}$ \& J. Muzerolle ${ }^{5}$
}

Magnetospheric accretion models predict that matter from protoplanetary disks accretes onto stars via funnel flows, which follow stellar magnetic field lines and shock on the stellar surfaces ${ }^{1-3}$, leaving hot spots with density gradients ${ }^{4-6}$. Previous work has provided observational evidence of varying density in hot spots ${ }^{7}$, but these observations were not sensitive to the radial density distribution. Attempts have been made to measure this distribution using X-ray observations ${ }^{8-10}$; however, X-ray emission traces only a fraction of the hot spot ${ }^{11,12}$ and also coronal emission ${ }^{13,14}$. Here we report periodic ultraviolet and optical light curves of the accreting star GM Aurigae, which have a time lag of about one day between their peaks. The periodicity arises because the source of the ultraviolet and optical emission moves into and out of view as it rotates along with the star. The time lag indicates a difference in the spatial distribution of ultraviolet and optical brightness over the stellar surface. Within the framework of a magnetospheric accretion model, this finding indicates the presence of a radial density gradient in a hot spot on the stellar surface, because regions of the hot spot with different densities have different temperatures and therefore emit radiation at different wavelengths.
We conducted a coordinated multiepoch multiwavelength observing campaign of the accreting T Tauri star GM Aur, a roughly 2-Myr-old solar analogue that is surrounded by a disk with a large cavity with a radius of about $20 \mathrm{AU}^{15-17}$. Gas is flowing inside the cavity and eventually reaches the star, as evidenced by moderate accretion rates $^{18}$; however, most of the solid material in the cavity has grown to millimetre and larger sizes, as indicated by the lack of substantial infrared emission ${ }^{15}$ that would be present if a substantial amount of smaller dust grains remained. Because giant planets must be present for large cavities (tens of astronomical units in size ${ }^{19,20}$ ) to open in protoplanetary disks, GM Aur is an ideal candidate to study a protoplanetary disk with the properties required to form planets.

Here we present results from our multiwavelength variability study, including Neil Gehrels Swift Observatory (Swift) X-ray and near-ultraviolet (NUV) fluxes, Hubble Space Telescope (HST) NUV spectra, Las Cumbres Observatory Global Telescope (LCOGT) $u^{\prime} g^{\prime} r^{\prime} i^{\prime}$ photometry, Transiting Exoplanet Survey Satellite (TESS) photometry and CHIRON H $\alpha$ spectra taken contemporaneously over roughly 35 days. We find no significant variation in the $X$-ray emission (Extended Data Fig. 1) and therefore rule it out as being responsible for any of the changes seen and do not discuss it further. Daily changes appear in the Swift NUV, LCOGT $u^{\prime} g^{\prime} r^{\prime} i^{\prime}$ and TESS light curves, with a period of about 6 days (Methods), consistent with the 6.1-day rotation period of the $\operatorname{star}^{21}$ and evidence of rotational modulation (Fig. 1). The NUV, $u^{\prime}$, and $g^{\prime}$ fluxes (Fig. 1, red shaded boxes) peak about a day before (Methods) the TESS, $r^{\prime}$ and $i^{\prime}$ fluxes (Fig. 1, green shaded boxes). Specifically, the UV data peaked on 2019 December 1, 7,13 (not as well defined owing to sparse data) and 19, whereas the optical data peaked on 2019 December 2, 8, 14 (not as well defined in the TESS light curve) and 20. There is also a dip in all the light curves on December 23-25 (Fig.1, grey shaded boxes). This is followed by the disappearance of the UV peaks in the light curves; the optical peaks remain, but appear to be lower than those before the dip in the light curves (Fig.1). We propose that this combination of features in the light curves is due to structure in the hot spot.

The time lag between the peaks in the UV and optical light curves and the eventual disappearance of the UV peaks is the first observational evidence of a density gradient in a hot spot on a stellar surface. The UV emission traces the high-density region of the hot spot and the optical emission traces the low-density region ${ }^{7}$. As the star rotates, we see the different density regions of the hot spot as it goes in and out of view. When the high-density region of the hot spot disappears, the UV emission decreases substantially.

Accretion-shock modelling confirms that the high-density region of the hot spot dominates the UV emission and that the low-density region of the hot spot dominates the optical emission (Methods). These models consist of a vertical accretion column close to the surface of the star that is constrained by the stellar magnetic field and divided into three subregions: the pre-shock zone, the post-shock zone and the heated photosphere below the shock ${ }^{22}$. The top of the accretion column is met by the funnel flow and the base is the hot spot on the stellar surface. There is a peak in both the observed UV emission and the model emission from the high-density region of the hot spot about 1 day

'Institute for Astrophysical Research, Department of Astronomy, Boston University, Boston, MA, USA. ²Department of Physics and Astronomy, Amherst College, Amherst, MA, USA.

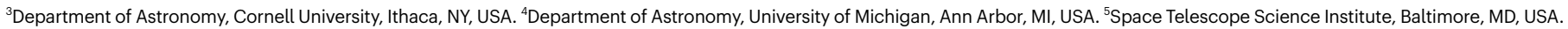
凶e-mail: cce@bu.edu 


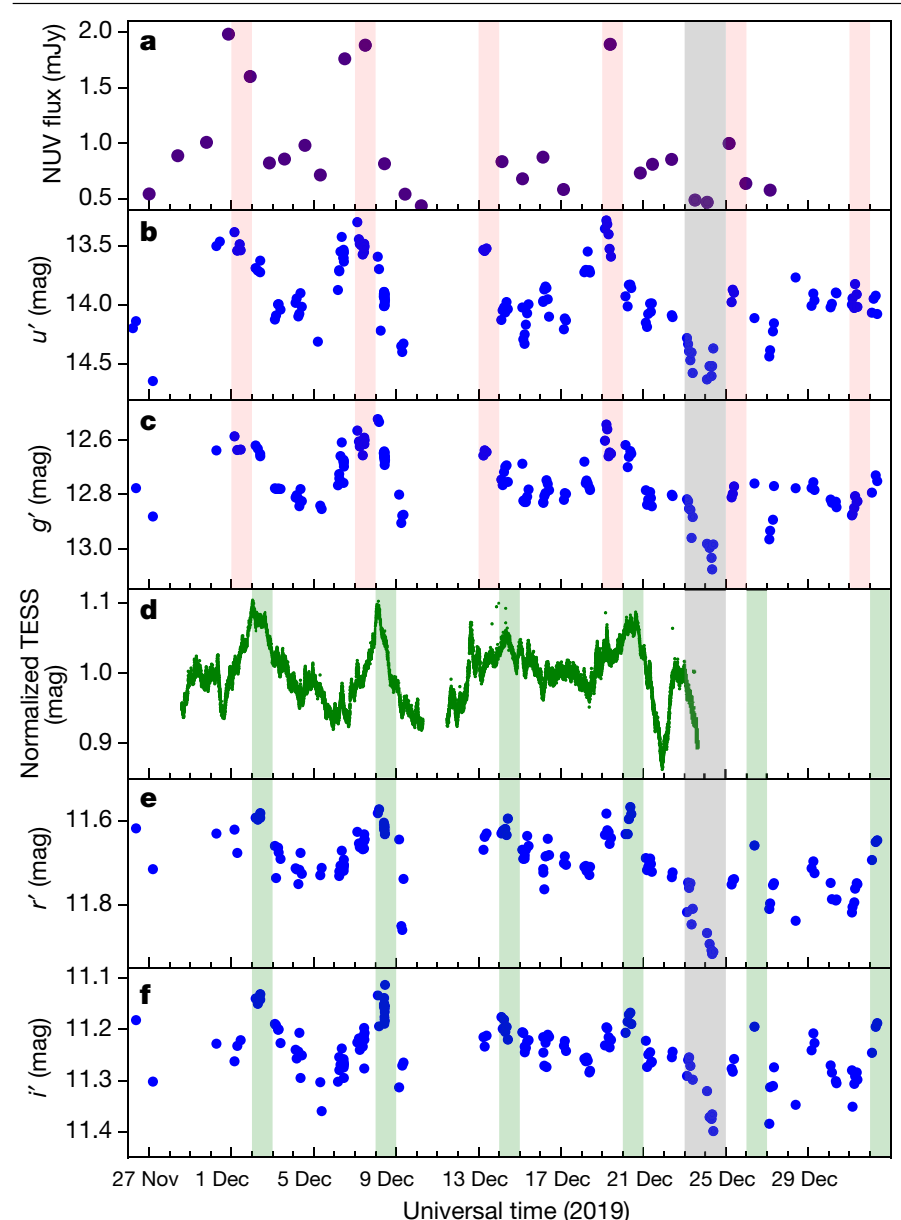

Fig. 1 Multiwavelength light curves of GM Aur. a-f, Swift NUV fluxes (a) Extended Data Table 1), LCOGT $u^{\prime} g^{\prime}$ flux calibrated magnitudes (b, c), TESS normalized magnitudes (d) and LCOGT $r^{\prime} \boldsymbol{i}^{\prime}$ flux calibrated magnitudes $(\mathbf{e}, \mathbf{f})$. The TESS bandpass covers the bandpass of the $r^{\prime}$ and $i^{\prime}$ data. Uncertainties are smaller than the symbols. Red and green shaded boxes highlight peaks in the UV and optical light curves, respectively, and are separated by the rotation period of the star. Dark grey shaded boxes highlight a contemporaneous dip in all the light curves.

before the peak in both the observed optical emission and the model emission from the low-density region (Fig. 2). Periodicity in the light curves (Methods) consistent with the stellar rotation period ${ }^{21}$ points to rotational modulation. This supports our contention that we are seeing a hot spot with a density gradient as it rotates along with the star.

The above is also consistent with magnetohydrodynamic simulations, which predict that there should be a density gradient in the accretion flow, column and hot $\operatorname{spot}^{5,8,23}$. We quantitatively compare the accretion-shock modelling results to the size of the hot spot predicted by three-dimensional magnetohydrodynamic models ${ }^{5}$. The low-density region of the hot spot is predicted to cover $10 \%-20 \%$ of the stellar surface. At high-density, this coverage can drop to less than $1 \%$. Our accretion-shock modelling shows that the surface coverage of the hot spot decreases at larger densities, consistent with the theoretical predictions. Specifically, the coverage of the low-density region is $10 \%-20 \%$ and the coverage of the high-density region is $0.1 \%$ (Fig. 2 , Extended Data Table 2, Methods). The sizes of these accretion hot spots are also roughly consistent with those measured from Zeeman Doppler imaging of young stars ${ }^{24}$.

We also qualitatively compare the observed light curves with three-dimensional magnetohydrodynamic simulations that have the approximate properties of GM Aur (Fig. 3, Methods). Our observations

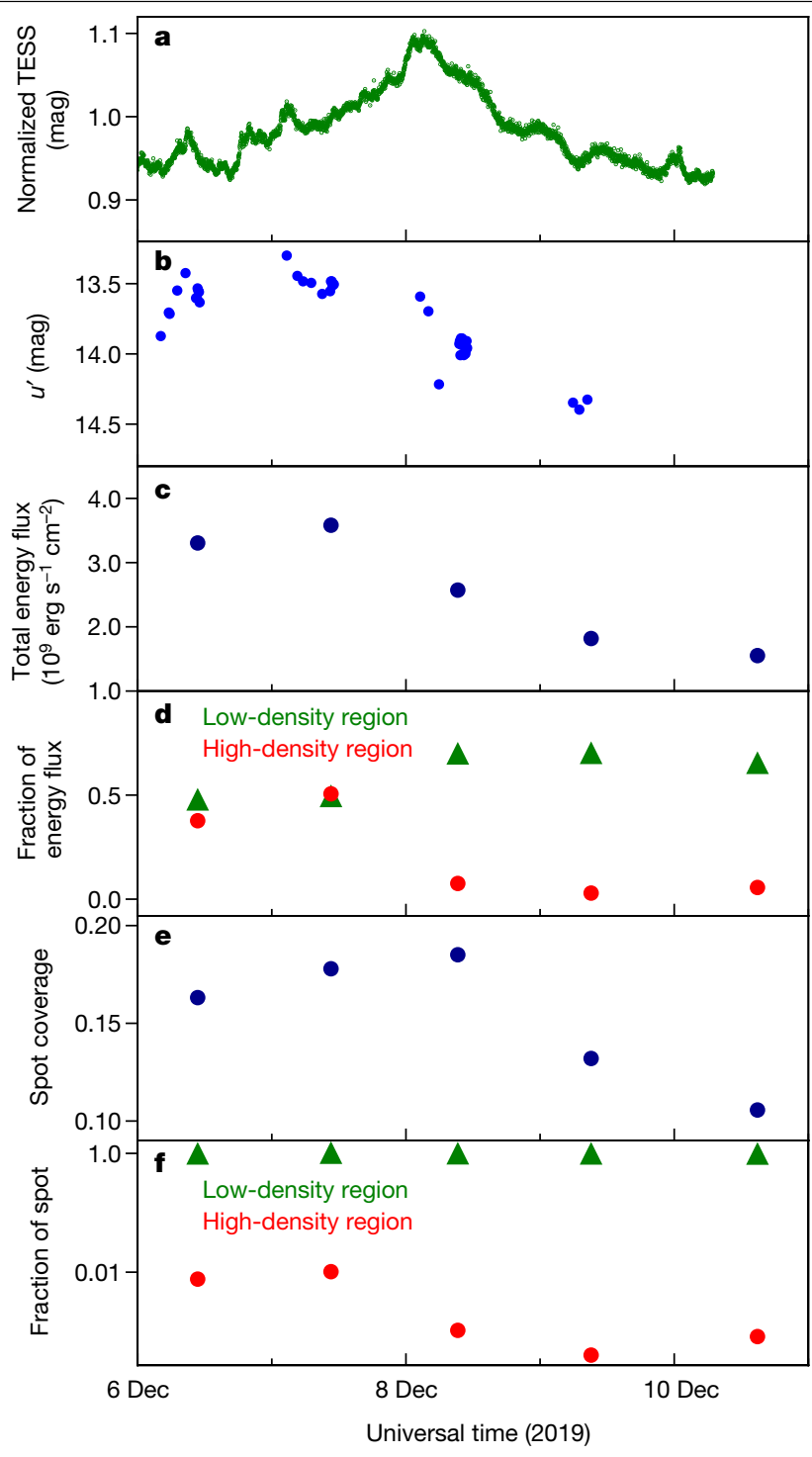

Fig. 2 | Comparison of simulated hot-spot properties to observed light curves. Best-fitting accretion-shock model parameters (Extended Data Table 2) are derived by fitting HST spectra (Methods). a, b, TESS optical light curve (a) and LCOGT $u^{\prime}$ light curve (b) between 2019 December 6 and 10. c, d, When $u^{\prime}$ peaks, the total energy flux of the accretion column (c) and the contribution from the high-density region (d) are highest. e, The size of the hot spot peaks along with the optical data.f, The low-density region dominates on all days, whereas the high-density region drops substantially after 2019 December 8.

point to a hot spot with a density gradient where at times the smaller high-density region of the hot spot is no longer visible (presumably because it is behind the star) while the larger low-density region is still visible. This is consistent with three-dimensional magnetohydrodynamic simulations ${ }^{5,23,25}$, which predict that there may be a density gradient in the hot spot and that at times parts of the hot spot may not be visible owing to stellar rotation (Fig. 4, top). The observations are also consistent with simulated light curves (Fig. 4, bottom). Like the observations, the simulated light curves are modulated mainly by the stellar rotation, and the emission from the high-density region of the spot peaks before the emission from the low-density region. Another piece of evidence of structure in the hot spot is the disappearance of the UV peaks in the light curves after December 25, while the optical peaks appear to remain (Fig. 1). The position, shape and structure of the hot spot varies with time, which particularly influences the high-density 


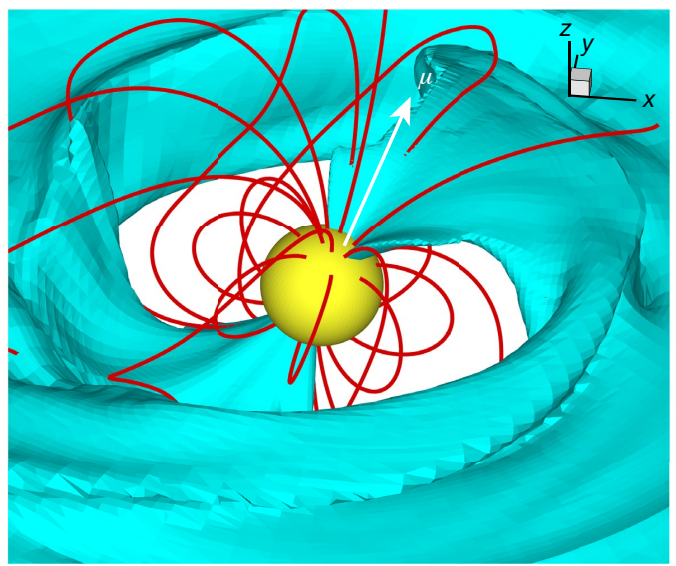

c

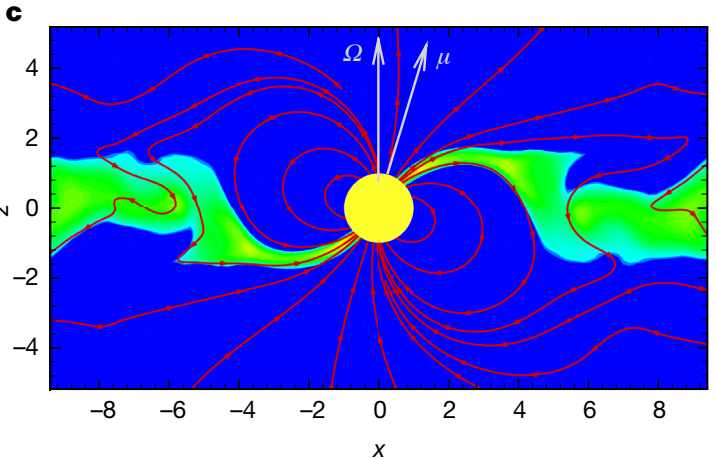

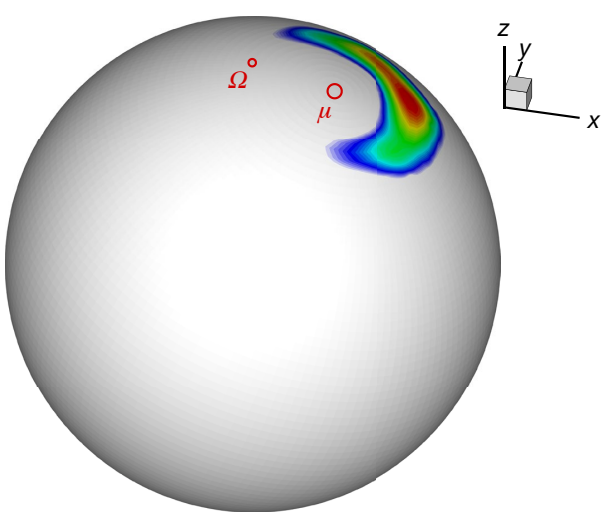

d

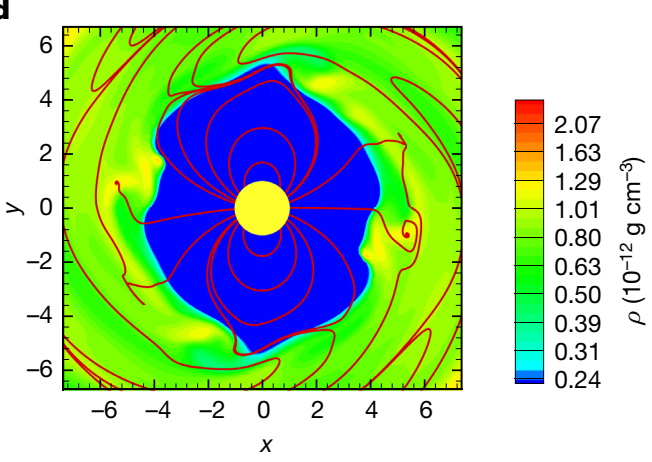

Fig. 3 | Three-dimensional magnetohydrodynamic simulations of accretion. a, A three-dimensional view of matter at a density level of $5.3 \times 10^{-13} \mathrm{~g} \mathrm{~cm}^{-3}$ (blue) flowing onto the star (yellow) along selected magnetic-field lines (red) at $3.13 P_{\text {star }}$ (where $P_{\text {star }}$ is the stellar rotation

region of the hot spot, causing it to occasionally disappear (Fig. 4, Methods). Therefore, emission from the low-density region of the hot spot persists while emission from the high-density region of the hot spot may disappear, as observed here.

The time delay between the peaks in the UV and optical data rules out the possibility that the hot spot is azimuthally symmetric. However, the simulations do not find that the hot spot is systematically asymmetric. Simulations show that the spots may become asymmetric owing to complex processes at the disk-magnetosphere boundary (Fig. 4, Methods). The observed systematic behaviour may result from a more complex multipole magnetic field closer to the star, which redirects part of the funnel flow such that the spots become systematically asymmetric in the azimuthal direction ${ }^{26,27}$. Previous three-dimensional simulations of stars with dipole and octupole fields show that if the octupole field dominates near the star it may redirect the flow and shape the hot $\operatorname{spot}^{28}$.

There are dips in the Swift, LCOGT and/or TESS light curves on December 21-22 and December 23-24 that precede the disappearance of the UV peaks. The first dip on December 21-22 is narrow and seen only in the TESS data (the Swift and LCOGT observations did not overlap in time). We see the start of the second dip on December 23 in the TESS data and we trace the entire dip in the Swift and LCOGT data (Fig. 1, dark grey shaded boxes). Dust extinction may lead to dips in the light curve ${ }^{29}$; however, GM Aur does not have substantial dust in the inner disk ${ }^{15}$. The observed dips are close to the expected minimum flux due to stellar rotation. There may have also been a decrease in accretion and hence hot-spot emission around this time, leading to a corresponding decrease at UV and optical wavelengths. This is consistent with the subsequent disappearance of the UV peaks and the lower optical peaks. period). Distance is measured in stellar radii $\left(R_{\mathrm{star}}\right)$. Only the central region $\left(42 R_{\text {star }}\right)$ is shown. b-d, The corresponding hot spot $(b)$, along with slices in the $x-z$ (c) and $x-y(\mathbf{d})$ planes; the colour scale denotes the density. Here $\mu$ is the magnetic moment, $\Omega$ is the rotation axis and $\rho$ is the density.

\section{Online content}

Any methods, additional references, Nature Research reporting summaries, source data, extended data, supplementary information, acknowledgements, peer review information; details of author contributions and competing interests; and statements of data and code availability are available at https://doi.org/10.1038/s41586-021-03751-5.

1. Hartmann, L., Herczeg, G. \& Calvet, N. Accretion onto pre-main-sequence stars. Annu. Rev. Astron. Astrophys. 54, 135-180 (2016).

2. Bouvier, J. et al. Probing the magnetospheric accretion region of the young pre-transitional disk system DoAr 44 using VLTI/GRAVITY. Astron. Astrophys. 636, A108 (2020).

3. GRAVITY Collaboration. A measure of the size of the magnetospheric accretion region in TW Hydrae. Nature 584, 547-550 (2020).

4. Romanova, M. M. \& Owocki, S. P. Accretion, outflows, and winds of magnetized stars. Space Sci. Rev. 191, 339-389 (2015).

5. Romanova, M. M. Ustyugova, G. V., Koldoba, A. V. \& Lovelace R. V. E. Three-dimensional simulations of disk accretion to an inclined dipole. II. Hot spots and variability. Astrophys. J. 610, 920-932 (2004)

6. Lamzin, S. A. The structure of shock waves in the case of accretion onto low-mass young stars. Astron. Rep. 42, 322-335 (1998).

7. Ingleby, L. et al. Accretion rates for T Tauri stars using nearly simultaneous ultraviolet and optical spectra. Astrophys. J. 767, 112 (2013).

8. Bonito, R. et al. Magnetohydrodynamic modeling of the accretion shocks in classical T Tauri stars: the role of local absorption in the X-ray emission. Astrophys. J. 795, L34 (2014).

9. Argiroffi, C. et al. Variable X-ray emission from the accretion shock in the classical T Tauri star V2129 Ophiuchi. Astron. Astrophys. 530, A1 (2011).

10. Schneider, P. C. et al. Multiepoch, multiwavelength study of accretion onto T Tauri. X-ray versus optical and UV accretion tracers. Astron. Astrophys. 618, A55 (2018).

11. Sacco, G. G. et al. On the Observability of T Tauri accretion shocks in the X-ray band. Astron. Astrophys. 522, A55 (2010).

12. Orlando, S. et al. X-ray emitting MHD accretion shocks in classical T Tauri stars. Case for moderate to high plasma-beta values. Astron. Astrophys. 510, A71 (2010).

13. Brickhouse, N. S. et al. A deep Chandra X-ray spectrum of the accreting young star TW Hydrae. Astrophys. J. 710, 1835-1847 (2010). 

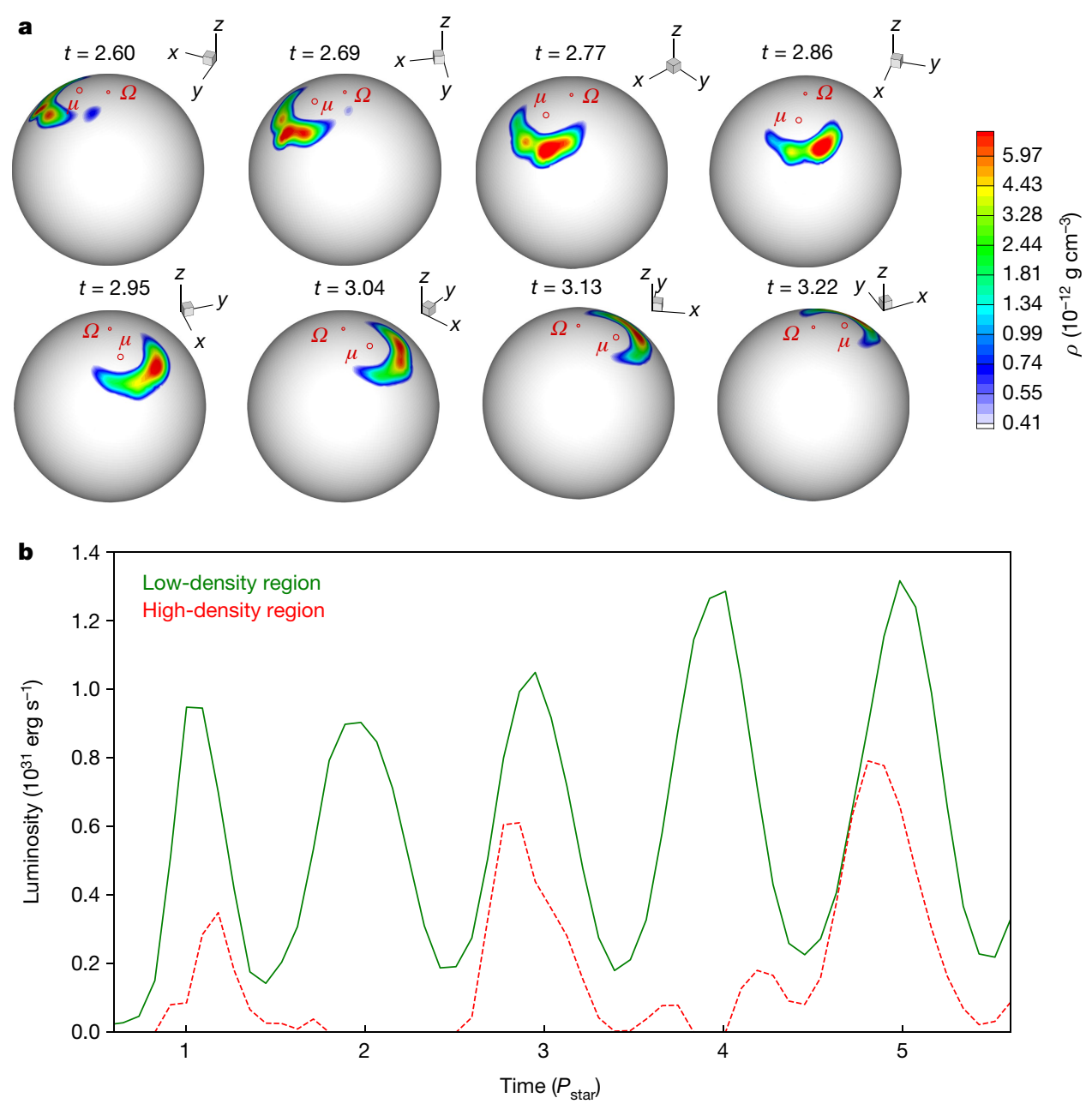

Fig. 4 | Simulations of the hot spot and light curves. a, The hot spot is crescent-shaped, is bent around the magnetic pole and has a density gradient. Here $\mu$ is the magnetic moment, $\Omega$ is the rotation axis, $\rho$ is the density and $t$ is time. $\mathbf{b}$, The light curves generated by the less dense (solid) and densest (broken) regions of the hot spot are modulated by the stellar rotation period $\left(P_{\text {star }}\right)$. The high-density region of the hot spot rotates out of view, leading to a time lag between the light curves at about $3 P_{\text {star }}$ and $5 P_{\text {star }}$; it may also occasionally disappear (at about $2 P_{\text {star }}$ and $4 P_{\text {star }}$ ).
14. Dupree, A. K. et al. TW Hya: spectral variability, $\mathrm{X}$-rays, and accretion diagnostics. Astrophys. J. 750, 73 (2012).

15. Calvet, N. et al. Disks in transition in the Taurus population: Spitzer IRS spectra of GM Aurigae and DM Tauri. Astrophys. J. 630, L185-L188 (2005).

16. Andrews, S. M. et al. Resolved images of large cavities in protoplanetary transition disks. Astrophys. J. 732, 42 (2011).

17. Macías, E. et al. Multiple rings in the transitional disk of GM Aurigae revealed by VLA and ALMA. Astrophys. J. 865, 37 (2018).

18. Robinson, C. E. \& Espaillat, C. C. Multiepoch ultraviolet HST observations of accreting low-mass stars. Astrophys. J. 874, 129 (2019)

19. Zhu, Z., Nelson, R. P., Hartmann, L., Espaillat, C. \& Calvet, N. Transitional and pre-transitional disks: gap opening by multiple planets? Astrophys. J. 729, 47 (2011).

20. Espaillat, C. et al. in Protostars and Planets VI (eds Beuther, H. et al.) 497-520 (Univ. Arizona Press, 2014).

21. Percy, J. R., Grynko, S., Seneviratne, R. \& Herbst, W. Self-correlation analysis of the photometric variability of T Tauri stars. II. A survey. Publ. Astron. Soc. Pacif. 122, 753-765 (2010).

22. Calvet, N. \& Gullbring, E. The structure and emission of the accretion shock in T Tauri stars. Astrophys. J. 509, 802-818 (1998).

23. Kulkarni, A. K. \& Romanova, M. M. Accretion to magnetized stars through the RayleighTaylor instability: global 3D simulations. Mon. Not. R. Astron. Soc. 386, 673-687 (2008).

24. Donati, J.-F. et al. Non-stationary dynamo and magnetospheric accretion processes of the classical T Tauri star V2129 Oph. Mon. Not. R. Astron. Soc. 412, 2454-2468 (2011).

25. Blinova, A. A., Romanova, M. M. \& Lovelace, R. V. E. Boundary between stable and unstable regimes of accretion. Ordered and chaotic unstable regimes. Mon. Not. $R$. Astron. Soc. 459, 2354-2369 (2016).
26. Donati, J.-F. et al. Magnetic fields and accretion flows on the classical T Tauri star V2129 Oph. Mon. Not. R. Astron. Soc. 380, 1297-1312 (2007).

27. Gregory, S. G., Matt, S. P., Donati, J.-F. \& Jardine, M. The non-dipolar magnetic fields of accreting T Tauri stars. Mon. Not. R. Astron. Soc. 389, 1839-1850 (2008).

28. Romanova, M. M., Long, M., Lamb, F. K., Kulkarni, A. K. \& Donati, J.-F. Global 3D simulations of disc accretion on to the classical T Tauri star V2129 Oph. Mon. Not. $R$. Astron. Soc. 411, 915-928 (2011).

29. Stauffer, J. et al. CSI 2264: characterizing young stars in NGC 2264 with short-duration periodic flux dips in their light curves. Astron. J. 149, 130 (2015).

Publisher's note Springer Nature remains neutral with regard to jurisdictional claims in published maps and institutional affiliations.

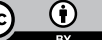

Open Access This article is licensed under a Creative Commons Attribution 4.0 International License, which permits use, sharing, adaptation, distribution and reproduction in any medium or format, as long as you give appropriate credit to the original author(s) and the source, provide a link to the Creative Commons license, and indicate if changes were made. The images or other third party material in this article are included in the article's Creative Commons license, unless indicated otherwise in a credit line to the material. If material is not included in the article's Creative Commons license and your intended use is not permitted by statutory regulation or exceeds the permitted use, you will need to obtain permission directly from the copyright holder. To view a copy of this license, visit http://creativecommons.org/licenses/by/4.0/.

(C) The Author(s) 2021 


\section{Methods}

\section{Observations and data reduction}

Our campaign of GM Aur was undertaken mostly during 2019 December. Here we present data spanning the X-ray to the optical from Swift, HST, LCOGT, TESS and CHIRON. We provide details on the data reduction below.

Swift observations were taken with the X-ray Telescope ${ }^{30}$ and the Ultraviolet and Optical Telescope ${ }^{31}$, using the UVM2 filter $(2,221 \AA)$, daily between 2019 November 27 and 2019 December 27, except on days when observations could not be scheduled. For the 27 observations, observation IDs, exposure times, start times, X-ray count rates, NUV fluxes and measurement uncertainties are listed in Extended Data Table 1. The observation IDs are as follows: 00342490450034249058, 0034249060-0034249063, 0034249065-0034249070 and 0034249072-0034249074. We used the High Energy Astrophysics Science Archive Research Center (HEASARC) HEASoft software (version 6.22.1) to measure count rates and NUV fluxes. X-ray emission from the stellar corona is known to be variable ${ }^{32,33}$. X-ray count rates $(0.5-10.0 \mathrm{keV})$ of GM Aur usually varied between 0.006 counts s $^{-1}$ and 0.029 counts s $^{-1}$ (Extended Data Fig. 1 ) and are comparable to those previously seen in GM Aur ${ }^{34}$, pointing to an X-ray luminosity of around $3 \times 10^{30} \mathrm{erg} \mathrm{s}^{-1}$ (assuming a distance of around $160 \mathrm{pc}^{35}$ ) throughout the campaign. The count rate was highest on 2019 November 27 $\left(0.046\right.$ counts $\left.\mathrm{s}^{-1}\right)$. None of the $\mathrm{X}$-ray variability observed seems to correlate with any other data obtained during this campaign (Fig. 1, Extended Data Fig. 1) and so we do not discuss it further.

HST NUV-near-infrared (NIR) spectra $(1,700-10,000 \AA$ with a resolution, $R$, of 500-1,000) were taken with the Space Telescope Imaging Spectrograph (STIS) on 2019 December 6, 7, 8, 9 and 10 (Extended Data Fig. 2) in programme 16010. The spectra were obtained with the G230L, G430L and G750 L gratings, with a $52 " \times 2$ " slit, within one orbit per visit. Data were reduced automatically by the HST pipeline; we correct fringing in the $\mathrm{G} 750 \mathrm{~L}$ data ${ }^{36}$. Archival G230L and G430L spectra of the non-accreting star RECX 1 (programme 11616) were used as the template for the accretion-shock modelling analysis below. Typical uncertainties on the HST spectra are $3 \%-15 \%$.

TESS data were taken on 2019 November 28 to 2019 December 23 (sector 19), with a cadence of $2 \mathrm{~min}$ (Fig. 1). The TESS bandpass covers $6,000-10,000 \AA$ and is centred at 7,865 $\AA$. Data were reduced using the Science Processing Operations Center pipeline. We used Lightkurve ${ }^{37}$ to check the data quality and found little contamination from nearby sources. The gap in the data is due to data download during orbital perigee. Typical uncertainties in the TESS data are $0.25 \%$.

$u^{\prime} g^{\prime} r^{\prime} i^{\prime}$ data were taken roughly $5-10$ times on clear nights at the LCOGT $^{38}$, with the Sinistro Imagers on the $1-m$ telescopes, between 2019 November 26 and 2020 January 1 . The $u^{\prime} g^{\prime} r^{\prime} i^{\prime}$ filters have central wavelengths of $3,540 \AA, 4,770 \AA, 6,215 \AA$ and $7,545 \AA$, and wavelength widths of $570 \AA, 1,500 \AA, 1,390 \AA$ and 1,290. . Data in Fig. 1 were reduced using the Aperture Photometry Tool and standard aperture photometry techniques. Uncertainties in all bands are less than 0.005 mag. The flux of GM Aur was calibrated using the fluxes of background objects, which in turn were flux-calibrated using UBVRI data of background objects (converted to $u^{\prime} g^{\prime} r^{\prime} i^{\prime 39}$ ) and the GD 64 standard field ${ }^{40}$. These calibration data were taken contemporaneously at the 4.3-m Lowell Discovery Telescope (LDT), using the Large Monolithic Imager, on 2019 December 2, 7, 10,13,18 and 21 . The LDT data are used here only to flux-calibrate the LCOGT data.

Medium-resolution $(R \approx 25,000)$ optical $(4,082-8,906 \AA)$ spectra were obtained with $\mathrm{CHIRON}^{41}$, on a $1.5-\mathrm{m}$ telescope that is part of the Small and Moderate Aperture Research Telescope System (SMARTS) at Cerro Tololo Inter-American Observatory, between 2019 November 28 and December 17 (Extended Data Fig. 3). The standard CHIRON pipeline is not optimized to extract the $\mathrm{H} \alpha$ profile of young stars; we reduce the spectra here ${ }^{42}$.

\section{Timing analysis}

Although the periodicity and time lag in the light curves are evident (Fig.1), here we measure the period of the TESS and LCOGT $u^{\prime} g^{\prime} r^{\prime} i^{\prime}$ light curves as well as the time lag between the peak in the $u^{\prime}$ light curve and those in the $g^{\prime} r^{\prime} i^{\prime}$ and TESS light curves.

The Swift NUV data are much sparser, and we do not analyse them in as much detail.

To measure the period in the light curves, we use the Astropy LombScargle periodogram function ${ }^{43-45}$. We obtain a 5.8-day period for the TESS light curve; for the $u^{\prime} g^{\prime} r^{\prime} i^{\prime}$ light curves, we measure periods of 6.3 , $6.3,6.3$ and 6.1 days. The Swift NUV flux is greater than $50 \%$ higher than surrounding days on November 30-December 1, December 7-8 and December 19 (Fig. 1), which is consistent with a roughly 6-day period. Given that the independently measured rotation period of the star is 6.1 days $^{21}$, we attribute the roughly 6 -day period measured in the light curves to stellar rotation.

For the time-lag analysis, we use the Python package Stingray ${ }^{46}$. When compared to the $u^{\prime}$ light curve, we measure time lags of around $0,0.75,1.1$ and 1 days for the $g^{\prime} r^{\prime} i^{\prime}$ and TESS light curves. The Swift NUV flux is highest on the same days as the peaks seen in the $u^{\prime}$ light curve (Fig.1). We conclude that the time lag between the UV and optical data is about 1 day.

\section{Accretion-shock modelling}

To further explore the roughly 1-day time lag in the peak between the UV and optical data seen in Fig. 1, we focus on 2019 December 6-10 (Fig. 2a, b) because HST data were taken daily during this time. HST NUV spectra are the best measure of accretion because the accretion column that channels material onto the surface of the star emits substantial energy at NUV wavelengths ${ }^{1}$.

The HST data were fitted using accretion-shock models ${ }^{22}$. These models provide information on the physical properties of the accretion column and the associated hot spot, which is the footprint of the accretion column on the stellar surface. The accretion column is characterized by an energy flux $\left(\rho v_{\mathrm{s}}^{3} / 2\right)$, which measures the density of material in the accretion column $(\rho)$, assuming that the magnetospheric radius $\left(R_{\text {mag }}\right)$ and infall velocity $\left(v_{\mathrm{s}}\right.$; here $456 \mathrm{~km} \mathrm{~s}^{-1}$, which depends on the stellar radius $R_{\text {star, }}$ stellar mass $M_{\text {star }}$ and $R_{\text {mag }}$ ) are constant. Each column has a filling factor $f$, which gives the fraction of the stellar surface covered by the column (that is, the hot spot).

GM Aur is a well studied source in the NUV, with eight previously modelled epochs ${ }^{18}$. In Fig. $2 \mathrm{c}-\mathrm{f}$, we plot the accretion-column model parameters (Extended Data Table 2) obtained from fitting the HST data (Extended Data Fig. 2). The fitting was done with three accretion columns ${ }^{7,18}$, with energy fluxes of $1 \times 10^{10} \mathrm{erg} \mathrm{s}^{-1} \mathrm{~cm}^{-2}, 1 \times 10^{11} \mathrm{erg} \mathrm{s}^{-1} \mathrm{~cm}^{-2}$ and $1 \times 10^{12} \mathrm{erg} \mathrm{s}^{-1} \mathrm{~cm}^{-2}$, and adopting published stellar parameters ${ }^{47}$. Here we refer to the $1 \times 10^{10} \mathrm{erg} \mathrm{s}^{-1} \mathrm{~cm}^{-2}$ and $1 \times 10^{12} \mathrm{erg} \mathrm{s}^{-1} \mathrm{~cm}^{-2}$ components as the 'low-density' and 'high-density' regions. The total energy flux ( $F_{\text {total }}$; Fig. 2c) is the sum of the energy fluxes of all the regions, weighted by their respective $f$. The total hot-spot coverage on the stellar surface (Fig. 2e) is the sum of the $f$ values for all the regions.

The total energy flux peaks along with the UV data (Fig. 2c). When the energy flux peaks on December 7, it is dominated by emission from a high-density region, which then drops substantially (Fig. 2d). This suggests that on December 7 we see the high-density region of the hot spot and that in the following three days most of this high-density region is no longer visible. Meanwhile, the hot-spot coverage of the star peaked about 1 day later, on December 8, along with the optical data (Fig. 2e), and the hot spot is dominated by emission from a low-density region (Fig. 2f). Because the low-density region of the accretion column emits its energy at longer wavelengths ${ }^{7}$, it follows that the optical emission peaks when the hot spot is at its largest. The observed behaviour in the light curves may be interpreted by combining two effects: the stellar rotation and the different physical locations for the high-density and 
low-density regions. It then follows that the high-density region of the hot spot (whose energy appears mainly in the UV) will lead to a peak in the UV data when the high-density parts of the hot spot are visible to the observer. As the star rotates, we may be seeing a denser part of the hot spot first, which then rotates out of view.

\section{Accretion-flow modelling}

To facilitate comparison to the three-dimensional (3D) magnetohydrodynamic (MHD) simulations, we need to estimate the magnetospheric radius of GM Aur. Magnetospheric accretion-flow modelling ${ }^{48-50}$ of the $\mathrm{H} \alpha$ line provides properties of the accretion flow. The model assumes that the magnetic, stellar-rotation and disk-rotation axes are aligned. The material flows onto the star along an axisymmetric accretion flow that arises from the co-rotating gas disk. The geometry of the flow is described by a dipolar magnetic field and characterized by an inner radius $\left(R_{\mathrm{i}}\right.$, which corresponds to $\left.R_{\text {mag }}\right)$ and the width of the flow $\left(W_{r}\right)$ at the disk plane. The model assumes a steady-flow prescription for a given accretion rate to determine the density at a given point. The temperature at each point is determined parametrically, scaled to the density assuming a constant heating rate in the flow; the maximum temperature in the flow $\left(T_{\max }\right)$ describes each model. To calculate the emission line profile, the model assumes the extended Sobolev approximation and calculates the mean intensity and the level population of a 16-level hydrogen atom, and uses the ray-by-ray method for a given viewing inclination $(i)$.

We created a large grid of models varying the accretion rate, $R_{\mathrm{mag}}, W_{r}$, $T_{\max }$ and $i$, using the ranges of parameters appropriate for accreting $\mathrm{T}$ Tauri stars ${ }^{49}$. With these combinations, we calculated around 72,000 model profiles. We convolved the model profiles with a Gaussian instrumental profile of CHIRON's resolution and fitted each observed profile inside $\pm 400 \mathrm{~km} \mathrm{~s}^{-1}$ from the line centre. The best fits are determined by calculating the $\chi^{2}$ for each combination of the model and observed profile.

For each observed profile, we selected 100 best fits and calculated the means of the accretion rate, $R_{\mathrm{mag}}, W_{r}, T_{\max }$ and $i$ (Extended Data Table 3 ). The accretion rates listed in Extended Data Table 3 are higher on the dates for which there are peaks in the UV emission. The derived $i$ are roughly consistent with the measured inclination of the disk, which is $53^{\circ 17} . W_{r}$ varies between $0.2 R_{\text {star }}$ and $0.5 R_{\text {star }} ; T_{\max }$ varies between $8,270 \mathrm{~K}$ and $9,120 \mathrm{~K}$. We use the derived $R_{\mathrm{mag}}$ of GM Aur to compare it to simulations in the next section.

In Extended Data Fig. 3, we show the best-fitting model to the $\mathrm{H} \alpha$ profiles. The fit to the line wings is very good. The regions with strong absorption features on the blue side of the line were excluded from the fit; this blueshifted absorption is probably from winds, which are not included in the model. There is no periodic pattern in the $\mathrm{H} \alpha$ line (Extended Data Fig. 3). However, multicomponent high-velocity blueshifted absorption, starting on December 7, occurs along with peaks in the UV emission (Fig. 1) and accretion rate (Extended Data Tables 2,3). There is a possible second component at higher, blueshifted velocity on December 6, which might imply a deceleration after the launching of a higher-density outflow event that may presage the accretion event on December 7. Models ${ }^{51}$ show that there is a disk-wind component to the H $\alpha$ profile, which manifests as blueshifted absorption; this component is dominated by the innermost disk, within tens of stellar radii.

\section{D MHD simulations of accretion}

Here we show global 3D MHD simulations of a rotating magnetized star accreting from a disk ${ }^{5,23,25}$. In brief, the models assume that the star has a dipole magnetic field with a misalignment angle $(\theta)$ between the rotation axis $(\Omega)$ and magnetic axis $(\mu)$. The rotation axes of the star and disk are aligned. Here we use simulations with the same set up as previous work ${ }^{23,25}$, with parameters chosen to approximate the properties of GM Aur.
We assume that the magnetic field of GM Aur can be approximated with a dipole field. The hot spot of GM Aur is at a high latitude of about $77^{\circ}$, measured from radial velocity variations of $\mathrm{He} \mathrm{I}(5,876 \AA)^{52}$; previous work also found that the hot spot is at high latitudes for dipoleoctupole configurations ${ }^{1}$. Also, GM Aur has a long rotation period ${ }^{21}$, and young stars with simpler, more dipole magnetic fields are slower rotators $^{53}$. Simulations ${ }^{23}$ show that the properties of the hot spot depend on $\theta$, the corotation radius $\left(R_{\mathrm{co}}\right)$ and $R_{\text {mag. }}$. For GM Aur, $\theta=13^{\circ 52}$. This is consistent with the range of inclinations of the accretion flow inferred from modelling the $\mathrm{H} \alpha$ profile (all the best-fitting inclinations in Extended Data Table 3 are within about $10^{\circ}$ of the system inclination of $\left.53^{\circ}\right)$. Using a stellar rotation period of 6.1 days $^{21}, R_{\text {star }}=2 R_{\text {sun }}$ and $M_{\text {star }}=1.36 M_{\text {sun }}{ }^{47}$ (where $R_{\text {sun }}$ and $M_{\text {sun }}$ are the radius and mass of the Sun), $R_{\text {co }}=7.8 R_{\text {star }}(0.07 \mathrm{AU})$. We measure a mean $R_{\text {mag }}$ of about $3.8 R_{\text {star }}$, with a range of $3.4 R_{\text {star }}$ to $4.6 R_{\text {star }}$ (Extended Data Table 3). The simulations use $\theta=20^{\circ}, R_{\mathrm{mag}} \approx 4.5 R_{\mathrm{star}}$ and $R_{\mathrm{co}}=5.7 R_{\mathrm{star}}$, which are consistent with the parameters of GM Aur.

The 3D MHD simulations have an approximate accretion rate of about $1.1 \times 10^{-8} M_{\text {sun }} \mathrm{yr}^{-1}$, which agrees with measurements from the accretion-shock and accretion-flow modelling (Extended Data Tables 2, 3). The energy distribution in the spots in the 3D MHD simulations varies between about $3.9 \times 10^{9} \mathrm{erg} \mathrm{s}^{-1} \mathrm{~cm}^{-2}$ and $1.2 \times 10^{11} \mathrm{erg} \mathrm{s}^{-1} \mathrm{~cm}^{-2}$; this is consistent with the accretion-shock models, which use energy fluxes of $1 \times 10^{10} \mathrm{erg} \mathrm{s}^{-1} \mathrm{~cm}^{-2}$ to $1 \times 10^{12} \mathrm{erg} \mathrm{s}^{-1} \mathrm{~cm}^{-2}$. The densities of the 3D MHD simulated spots in Fig. 4 range between about $5.5 \times 10^{-13} \mathrm{~g} \mathrm{~cm}^{-3}$ and $5.97 \times 10^{-12} \mathrm{~g} \mathrm{~cm}^{-3}$, which overlaps with the accretion-shock models, where the densities are $2.1 \times 10^{-13} \mathrm{~g} \mathrm{~cm}^{-3}$ to $2.1 \times 10^{-11} \mathrm{~g} \mathrm{~cm}^{-3}$. The simulated light curves peak at a luminosity of about $1 \times 10^{31} \mathrm{erg} \mathrm{s}^{-1}$ (Fig. 4), which is roughly consistent with the accretion luminosities of about $0.1 L_{\text {sun }}$ to $0.2 L_{\text {sun }}\left(3.8 \times 10^{32} \mathrm{erg} \mathrm{s}^{-1}\right.$ to $7.7 \times 10^{32} \mathrm{erg} \mathrm{s}^{-1} ; L_{\text {sun }}$ is the luminosity of the Sun) measured from the HST spectra. In Fig. 4 we show the light curve only once accretion onto the star begins in the simulation. To generate the light curve for the densest region of the hot spot, we use $\rho>6.5 \times 10^{-12} \mathrm{~g} \mathrm{~cm}^{-3}$.

A ratio of $R_{\mathrm{co}} / R_{\mathrm{mag}}=1.5$ sets the boundary between stable and unstable accretion ${ }^{25}$. In the stable regime, matter accretes onto the star in ordered funnel streams, and symmetric, crescent-shaped hot spots are expected ${ }^{54}$. In the unstable regime, matter accretes in chaotic hot spots $^{25}$. The simulations have $R_{\mathrm{co}} / R_{\mathrm{mag}}=1.3-1.4$. The measured $R_{\mathrm{mag}}$ of GM Aur has an uncertainty of about $1 R_{\mathrm{star}}$, so $R_{\mathrm{co}} / R_{\mathrm{mag}}$ for GM Aur could reach 1.4. Therefore, the simulations are consistent with the properties of GM Aur, and both are near the boundary of the stable-unstable regime. Here, processes at the disk and magnetosphere boundary may cause the behaviour seen in the observed light curves. The inner disk rotates more rapidly than the magnetosphere, leading to non-stationary behaviour of matter in the inner disk and corresponding non-stationary behaviour of the hot spot. The hot spots are predominantly crescent-shaped, but may become asymmetric (Fig. 4). Also resulting from the difference in the disk and magnetosphere rotation, the non-stationarity leads to variation of the density distribution in the hot spot that especially influences the densest parts of the spot, which may occasionally disappear. This may explain why the UV emission (associated with the high-density region of the hot spot) disappears in Fig. 1.

\section{Data availability}

The raw data for the Swift, HST, TESS (https://archive.stsci.edu) and LCOGT (https://lco.global/) data are publicly available from their respective archives. The LDT and CHIRON raw data are available on request from C.C.E. HST and TESS provide reduced data in their archives. Reduced Swift, LCOGT, CHIRON and LDT data are available on request from C.C.E. Data from the accretion-shock and accretion-flow modelling are available on request from C.C.E. Data relevant to the 3D MHD numerical simulations are available on request from M.M.R. (romanova@astro.cornell.edu). 


\section{Code availability}

Access to the accretion-shock, accretion-flow and MHD model codes are available on request from N.C. (ncalvet@umich.edu),J.M. (muzerol@ stsci.edu) and M.M.R., respectively.

30. Burrows, D. N. et al. The Swift X-ray telescope. Space Sci. Rev. 120, 165-195 (2005).

31. Roming, P. W. A. et al. The Swift ultra-violet/optical telescope. Space Sci. Rev. 120, 95-142 (2005).

32. Preibisch, T. et al. The origin of T Tauri X-ray emission: new insights from the Chandra Orion ultradeep project. Astrophys. J. Suppl. Ser. 160, 401-422 (2005).

33. Guarcello, M. G. et al. CSI 2264: simultaneous optical and X-ray variability in pre-main sequence stars. I. Time resolved X-ray spectral analysis during optical dips and accretion bursts in stars with disks. Astron. Astrophys. 602, A10 (2017).

34. Espaillat, C. C., Robinson, C., Grant, S. \& Reynolds, M. Using multiwavelength variability to explore the connection among X-ray emission, the far-ultraviolet $\mathrm{H}_{2}$ bump, and accretion in T Tauri stars. Astrophys. J. 876, 121 (2019).

35. Gaia Collaboration. Gaia data release 2. Summary of the contents and survey properties. Astron. Astrophys. 616, A1 (2018).

36. Goudfrooij, P., \& Christensen, J. A. STIS Near-IR Fringing. III. A Tutorial on the Use of the IRAF Tasks. Report No. 98-29 (STIS Instrument Science, 1998).

37. Lightkurve Collaboration. Lightkurve: Kepler and TESS time series analysis in Python. Astrophysics Source Code Library 1812.013 (2018).

38. Brown, T. M. et al. Las Cumbres Observatory global telescope network. Publ. Astron. Soc. Pacif. 125, 1031 (2013).

39. Jester, S. et al. The Sloan Digital Sky Survey view of the Palomar-Green bright quasar survey. Astron. J. 130, 873-895 (2005).

40. Landolt, A. U. UBVRI photometric standard stars around the sky at +50 deg declination. Astron. J. 146, 131 (2013).

41. Schwab, C., Spronck, J. F. P., Tokovinin, A. \& Fischer, D. A. Design of the CHIRON high-resolution spectrometer at CTIO. Proc. SPIE 7735, 77354G (2010).

42. .Walter, F. An Alternative Scheme for Chiron Fiber and Slicer Mode Data Reductions http:// www.astro.sunysb.edu/fwalter/SMARTS/CHIRON/ch reduce.pdf (2008).

43. Astropy Collaboration. The Astropy project: building an open-science project and status of the v2.0 core package. Astron. J. 156, 123 (2018).

44. Lomb, N. R. Least-squares frequency analysis of unequally spaced data. Astrophys. Space Sci. 39, 447-462 (1976)

45. Scargle, J. D. Studies in astronomical time series analysis. II. Statistical aspects of spectral analysis of unevenly spaced data. Astrophys. J. 263, 835-853 (1982).

46. Huppenkothen, D. et al. Stingray: a modern Python library for spectral timing. Astrophys J. 881, 39 (2019).
47. Manara, C. F. et al. Gas content of transitional disks: a VLT/X-Shooter study of accretion and winds. Astron. Astrophys. 568, A18 (2014).

48. Hartmann, L., Hewett, R. \& Calvet, N. Magnetospheric accretion models for T Tauri stars. I. Balmer line profiles without rotation. Astrophys. J. 426, 669-687 (1994).

49. Muzerolle, J., Calvet, N. \& Hartmann, L. Magnetospheric accretion models for the hydrogen emission lines of T Tauri stars. Astrophys. J. 492, 743-753 (1998).

50. Muzerolle, J., Calvet, N. \& Hartmann, L. Emission-line diagnostics of T Tauri magnetospheric accretion. II. Improved model tests and insights into accretion physics. Astrophys. J. 550, 944-961 (2001).

51. Lima, G. H. R. A., Alencar, S. H. P., Calvet, N., Hartmann, L. \& Muzerolle, J. Modeling the Ha line emission around classical T Tauri stars using magnetospheric accretion and disk wind models. Astron. Astrophys. 522, A104 (2010).

52. McGinnis, P., Bouvier, J. \& Gallet, F. The magnetic obliquity of accreting T Tauri stars. Mon. Not. R. Astron. Soc. 497, 2142-2162 (2020).

53. Johnstone, C. P. et al. Tauri stars: magnetic fields, coronae, and star-disc interactions. Mon. Not. R. Astron. Soc. 437, 3202-3220 (2014).

54. Kulkarni, A. K. \& Romanova, M. M. Analytical hotspot shapes and magnetospheric radius from 3D simulations of magnetospheric accretion. Mon. Not. R. Astron. Soc. 433, 30483061 (2013).

Acknowledgements C.C.E. acknowledges support from HST grant GO-16010, NASA grant 80NSSC19K1712 and NSF career grant AST-1455042. M.M.R. acknowledges the NSF grant AST20009820. N.C. acknowledges support from NASA grant NNX17AE57G.

Author contributions C.C.E. planned the observing campaign, analysed and interpreted the data, and wrote the manuscript. C.E.R. reduced the HST, TESS and CHIRON data, provided related text and did the HST data modelling. M.M.R. did the 3D MHD simulations, provided related text and aided in the interpretation of the data. T.T. did the CHIRON data modelling and provided related text. J.W. reduced the LCOGT and LDT data and did the timing analysis. N.C. aided in the interpretation of the data. M.R. reduced the Swift data. J.M. aided in the interpretation of the CHIRON data. All authors participated in the discussion of results and revision of the manuscript.

Competing interests The authors declare no competing interests.

\section{Additional information}

Supplementary information The online version contains supplementary material available at https://doi.org/10.1038/s41586-021-03751-5.

Correspondence and requests for materials should be addressed to C.C.E.

Peer review information Nature thanks Hans Moritz Guenther and the other, anonymous,

reviewer(s) for their contribution to the peer review of this work.

Reprints and permissions information is available at http://www.nature.com/reprints. 


\section{Article}

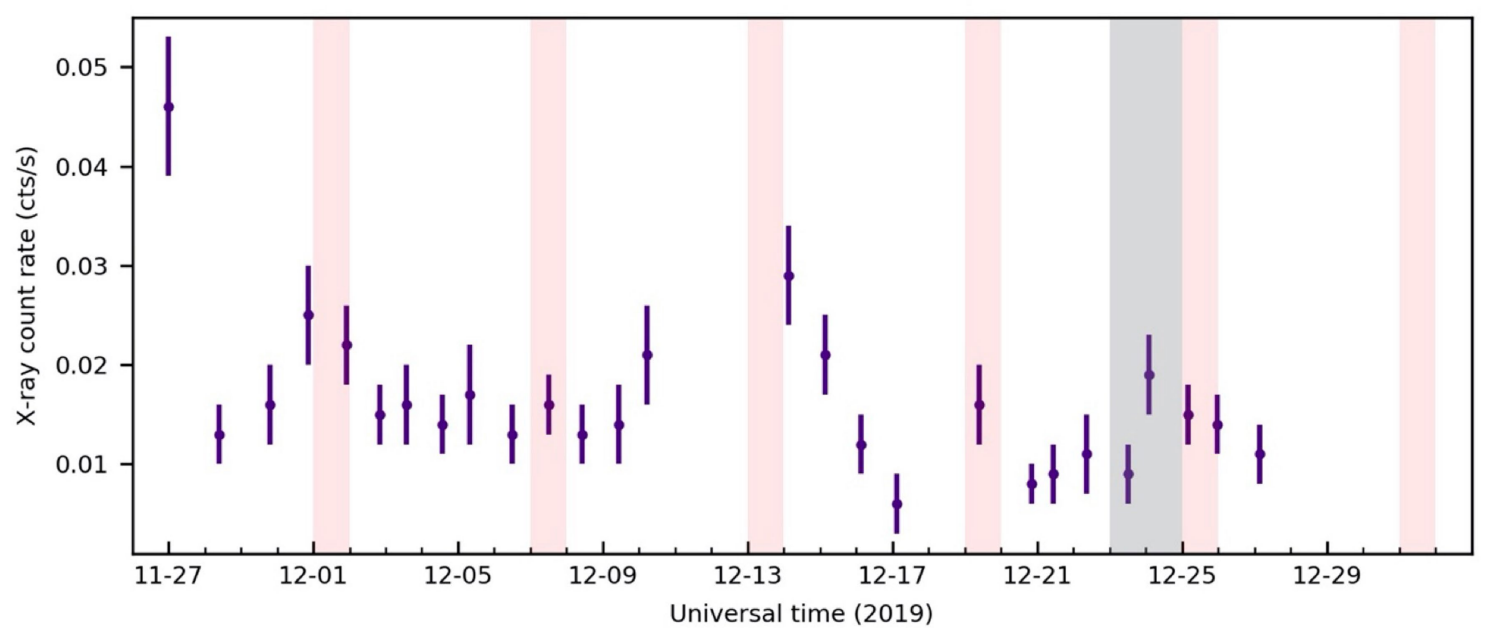

Extended Data Fig. 1 |X-ray count rates of GM Aur. Data were observed by bars as in Fig. 1 for the Swift NUV data. Error bars correspond to uncertainties, Swift between 2019 November 26 and 2020 January 1, and are listed in Extended which are listed in Extended Data Table 1. Data Table1. To facilitate comparison, we use the same time range and colour 


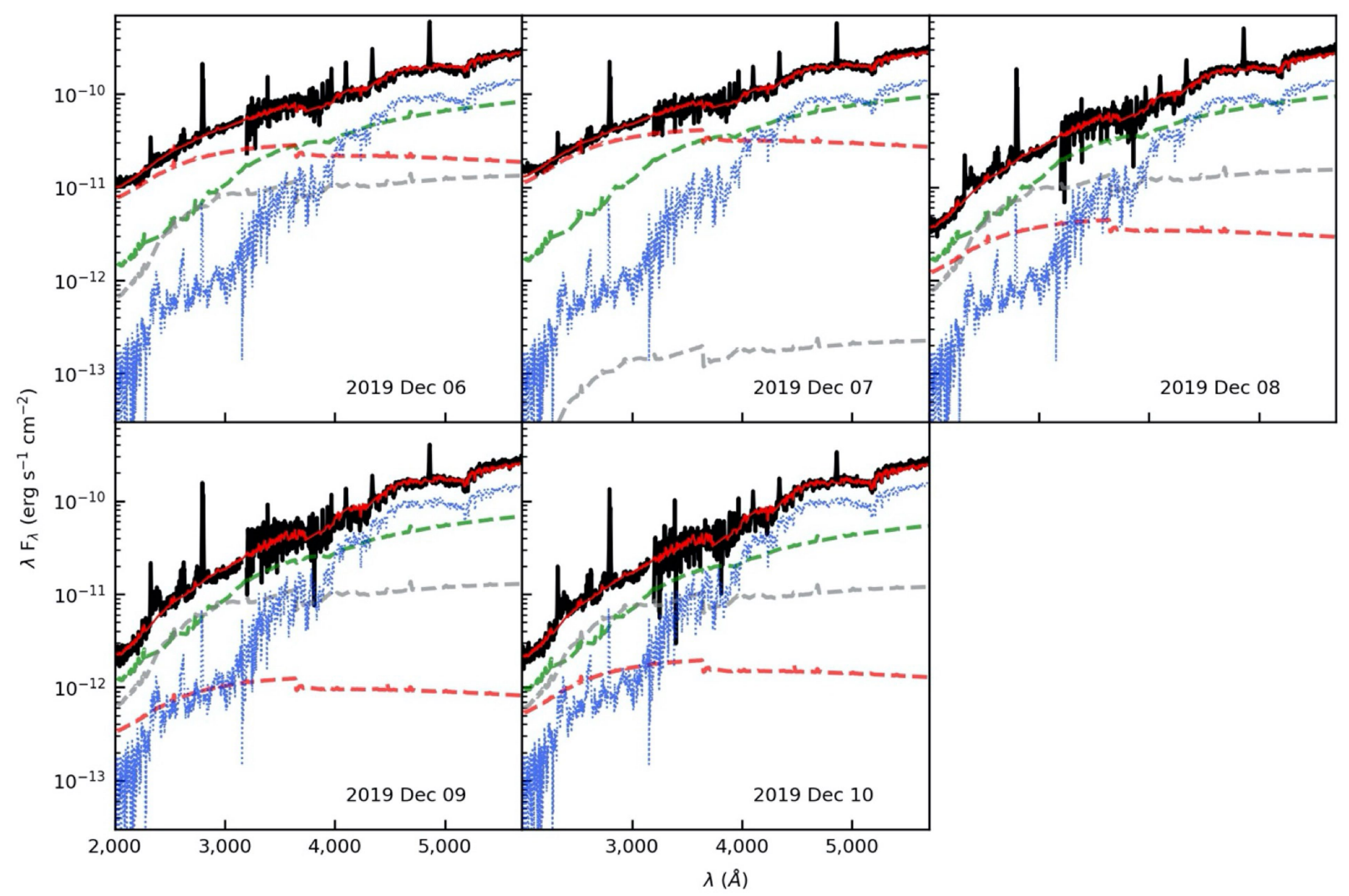

Extended Data Fig. 2 | Accretion shock modelling. We show the best-fitting models (red solid line) to HST NUV-NIR spectra (black solid line). The total $1 \times 10^{11} \mathrm{erg} \mathrm{s}^{-1} \mathrm{~cm}^{-2}$ (grey dashed line) and $1 \times 10^{12} \mathrm{erg} \mathrm{s}^{-1} \mathrm{~cm}^{-2}$ (red dashed line). Best-fitting model parameters are listed in Extended Data Table 2. $\lambda$ and $F_{\lambda}$ are model consists of a non-accreting template star (blue dotted line) and three the wavelength and flux density, respectively. 


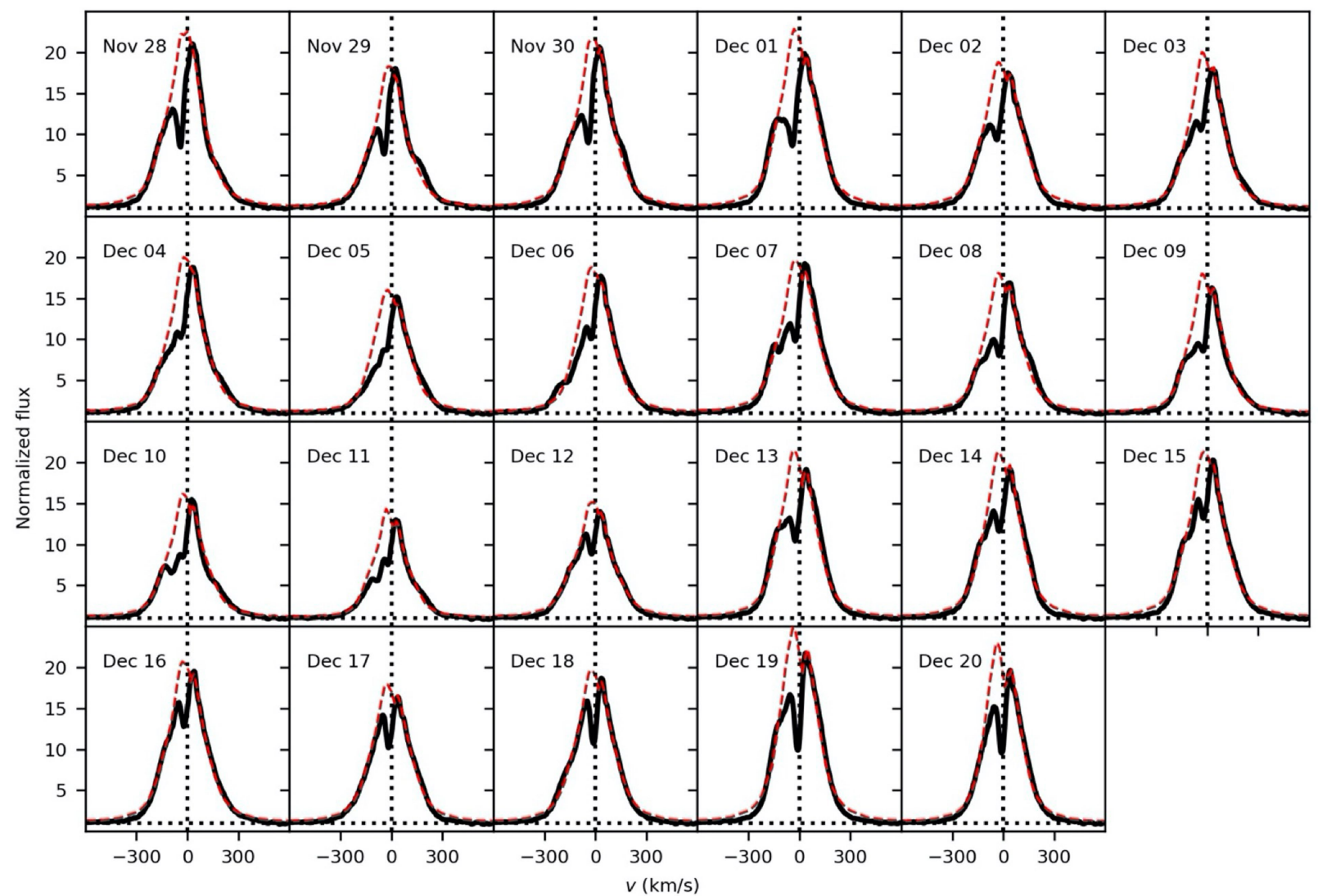

Extended Data Fig. 3 | Accretion flow modelling. We show the best-fitting models (red dashed lines) to $\mathrm{H} \alpha$ line profiles (black solid lines) measured with CHIRON and plotted in velocity, $v$. Best-fitting model parameters are listed in
Extended Data Table 3. There is evidence for a wind that is absorbing part of the $\mathrm{H} \alpha$ line profile on the blue side. 
Extended Data Table 1 | Swift observations and fluxes

\begin{tabular}{|c|c|c|c|c|}
\hline Obs. ID & Exp. time (s) & Start time (UT) & $\begin{array}{l}\text { X-ray count rates } \\
\text { (cts/s) }\end{array}$ & NUV flux (mJy) \\
\hline 00034249045 & 988.92704 & 2019-11-27Т00:12:19 & $0.046 \pm 0.007$ & $0.547 \pm 0.016$ \\
\hline 00034249046 & 1308.57631 & 2019-11-28T09:33:00 & $0.013 \pm 0.003$ & $0.890 \pm 0.022$ \\
\hline 00034249047 & 1278.60907 & 2019-11-29T19:00:29 & $0.016 \pm 0.004$ & $1.010 \pm 0.025$ \\
\hline 00034249048 & 1138.76270 & 2019-11-30Т20:32:49 & $0.025 \pm 0.005$ & $1.981 \pm 0.046$ \\
\hline 00034249049 & 1545.81653 & 2019-12-01T21:58:58 & $0.022 \pm 0.004$ & $1.601 \pm 0.037$ \\
\hline 00034249050 & 1530.83276 & 2019-12-02T20:18:17 & $0.015 \pm 0.003$ & $0.825 \pm 0.020$ \\
\hline 00034249051 & 1086.31981 & 2019-12-03Т13:52:42 & $0.016 \pm 0.004$ & $0.860 \pm 0.022$ \\
\hline 00034249052 & 1333.54882 & 2019-12-04T13:42:35 & $0.014 \pm 0.003$ & $0.983 \pm 0.024$ \\
\hline 00034249053 & 659.28785 & 2019-12-05T07:37:49 & $0.017 \pm 0.005$ & $0.717 \pm 0.022$ \\
\hline 00034249054 & 1428.44487 & 2019-12-06T12:04:59 & $0.013 \pm 0.003$ & $1.760 \pm 0.040$ \\
\hline 00034249055 & 1443.42813 & 2019-12-07T11:57:44 & $0.016 \pm 0.003$ & $1.882 \pm 0.043$ \\
\hline 00034249056 & 1238.65342 & 2019-12-08T10:22:09 & $0.013 \pm 0.003$ & $0.818 \pm 0.021$ \\
\hline 00034249057 & 1146.25422 & 2019-12-09T10:17:41 & $0.014 \pm 0.004$ & $0.545 \pm 0.015$ \\
\hline 00034249058 & 998.91566 & 2019-12-10T05:14:08 & $0.021 \pm 0.005$ & $0.441 \pm 0.014$ \\
\hline 00034249060 & 1395.99066 & 2019-12-14T03:16:01 & $0.029 \pm 0.005$ & $0.837 \pm 0.021$ \\
\hline 00034249061 & 1493.38366 & 2019-12-15Т03:08:33 & $0.021 \pm 0.004$ & $0.683 \pm 0.018$ \\
\hline 00034249062 & 1475.90302 & 2019-12-16Т02:58:31 & $0.012 \pm 0.003$ & $0.877 \pm 0.022$ \\
\hline 00034249063 & 941.47858 & 2019-12-17T02:53:06 & $0.006 \pm 0.003$ & $0.587 \pm 0.017$ \\
\hline 00034249065 & 1216.17749 & 2019-12-19T09:16:32 & $0.016 \pm 0.004$ & $1.890 \pm 0.044$ \\
\hline 00034249066 & 1328.55418 & 2019-12-20T20:21:19 & $0.008 \pm 0.002$ & $0.735 \pm 0.019$ \\
\hline 00034249067 & 1313.57088 & 2019-12-21T10:31:53 & $0.009 \pm 0.003$ & $0.813 \pm 0.021$ \\
\hline 00034249068 & 906.52676 & 2019-12-22T08:48:54 & $0.011 \pm 0.004$ & $0.857 \pm 0.023$ \\
\hline 00034249069 & 1470.89856 & 2019-12-23T12:04:16 & $0.009 \pm 0.003$ & $0.492 \pm 0.013$ \\
\hline 00034249070 & 1073.84346 & 2019-12-24T02:16:51 & $0.019 \pm 0.004$ & $0.472 \pm 0.014$ \\
\hline 00034249072 & 1440.94136 & 2019-12-25T03:45:28 & $0.015 \pm 0.003$ & $1.000 \pm 0.024$ \\
\hline 00034249073 & 1253.64681 & 2019-12-25T23:18:34 & $0.014 \pm 0.003$ & $0.641 \pm 0.017$ \\
\hline 00034249074 & 1398.48779 & 2019-12-27Т03:35:31 & $0.011 \pm 0.003$ & $0.581 \pm 0.016$ \\
\hline
\end{tabular}




\section{Article}

Extended Data Table 2 | Accretion-shock model parameters

\begin{tabular}{ccccccc}
\hline Date & $f\left(1 \times 10^{10}\right)$ & $f\left(1 \times 10^{11}\right)$ & $f\left(1 \times 10^{12}\right)$ & $\begin{array}{c}\mathrm{F}_{\text {total }} \\
\left(\mathrm{erg} \mathrm{s}^{-1} \mathrm{~cm}^{-2}\right)\end{array}$ & $f_{\text {total }}$ & $\begin{array}{c}\text { Accretion rate } \\
\left(10^{-8} \mathrm{M}_{\text {sun }} / \mathrm{yr}\right)\end{array}$ \\
\hline $12-6$ & 0.157 & 0.005 & 0.001 & $3.3 \times 10^{9}$ & 0.163 & 1.2 \\
$12-7$ & 0.176 & $9 \times 10^{-5}$ & 0.002 & $3.6 \times 10^{9}$ & 0.178 & 1.3 \\
$12-8$ & 0.179 & 0.006 & $1.9 \times 10^{-4}$ & $2.6 \times 10^{9}$ & 0.185 & 0.94 \\
$12-9$ & 0.127 & 0.005 & $5.3 \times 10^{-5}$ & $1.8 \times 10^{9}$ & 0.132 & 0.67 \\
$12-10$ & 0.101 & 0.005 & $8.7 \times 10^{-5}$ & $1.6 \times 10^{9}$ & 0.106 & 0.57 \\
\hline
\end{tabular}


Extended Data Table 3| Accretion-flow model parameters

\begin{tabular}{cccccc}
\hline $\begin{array}{c}\text { Date } \\
(2019 \text { UT })\end{array}$ & $\begin{array}{c}\text { Accretion rate } \\
\left(10^{-8} M_{\text {sun }} / \mathrm{yr}\right)\end{array}$ & $\begin{array}{c}\mathrm{R}_{\text {mag }} \\
\left(\mathrm{R}_{\text {star }}\right)\end{array}$ & $\begin{array}{c}\mathrm{W}_{\mathrm{r}} \\
\left(\mathrm{R}_{\text {star }}\right)\end{array}$ & $\begin{array}{c}\mathrm{T}_{\max } \\
(\mathrm{K})\end{array}$ & $\begin{array}{c}i \\
())\end{array}$ \\
\hline $11-28$ & 1.1 & 4.6 & 0.3 & 9060 & 65.6 \\
$11-29$ & 1.0 & 3.8 & 0.2 & 8960 & 58.4 \\
$11-30$ & 1.2 & 4.2 & 0.2 & 9120 & 60.4 \\
$12-01$ & 1.6 & 4.1 & 0.3 & 8880 & 53.4 \\
$12-02$ & 1.3 & 3.6 & 0.2 & 9010 & 48.1 \\
$12-03$ & 1.3 & 3.7 & 0.2 & 8850 & 49.8 \\
$12-04$ & 1.1 & 3.8 & 0.2 & 8910 & 52.6 \\
$12-05$ & 1.2 & 3.4 & 0.2 & 8600 & 45.6 \\
$12-06$ & 1.4 & 3.6 & 0.2 & 8680 & 47.1 \\
$12-07$ & 1.4 & 3.7 & 0.2 & 8800 & 49.4 \\
$12-08$ & 1.2 & 3.5 & 0.2 & 8870 & 48.2 \\
$12-09$ & 1.1 & 3.9 & 0.2 & 8500 & 53.9 \\
$12-10$ & 1.0 & 3.8 & 0.2 & 8750 & 61.1 \\
$12-11$ & 1.2 & 3.5 & 0.2 & 8270 & 51.7 \\
$12-12$ & 1.2 & 3.6 & 0.3 & 8880 & 60.2 \\
$12-13$ & 1.5 & 4.0 & 0.4 & 8800 & 49.9 \\
$12-14$ & 1.6 & 3.9 & 0.3 & 8760 & 48.3 \\
$12-15$ & 1.3 & 4.0 & 0.2 & 8910 & 53.7 \\
$12-16$ & 1.2 & 3.9 & 0.3 & 9010 & 52.2 \\
$12-17$ & 1.4 & 3.5 & 0.3 & 8920 & 48.0 \\
$12-18$ & 1.6 & 3.9 & 0.3 & 8700 & 53.5 \\
$12-19$ & 1.9 & 4.2 & 0.5 & 8630 & 45.1 \\
$12-20$ & 1.7 & 3.9 & 0.3 & 8490 & 43.6 \\
\hline
\end{tabular}

UT, universal time. 